\title{
Analysis of Platelet Refractoriness in a Tertiary Care Hospital
}

\author{
Jena PS, Sahoo D*, Acharya DP, Pati B, Kanungo GN and Behera R \\ Department of Transfusion Medicine, IMS \& SUM Hospital, India
}

*Corresponding author: Dibyajyoti Sahoo, Department of Transfusion Medicine, IMS \& SUM Hospital, India, Tel: 9040508970; Email: dib.jit@gmail.com

Research Article

Volume 4 Issue 1

Received Date: April 08, 2020

Published Date: April 24, 2020

DOI: $10.23880 /$ hij-16000158

\section{Abstract}

Introduction: Platelet refractoriness is a major concern for treating physicians. Refractoriness is also aggravated by a number co-existing conditions such as fever, sepsis, splenomegaly, drug intake, chemotherapy etc. Refractoriness may also be due to immunological causes such as HLA (Human Leukocyte Antigen) antibodies and anti-platelet antibodies.

Material and Methods: This study was prospective observational study undertaken in the department of Transfusion Medicine of the Institute of Medical Sciences and SUM hospital, Bhubaneswar India. The study population included patients who required transfusion of platelets by SDP (Single Donor Platelet) concentrates. The study group included patients of both sexes and of all age groups. Data was collected from patient, patient register present in the treating units and analysed.

Results: Out of the 155 patients 93 patients received single unit of SDP transfusions and 62 patients received two or more units of SDP. Out of the 62 patients, 28 patients received two SDP transfusions, 17 patients received 3 SDP transfusions, 6 patients received 4 SDP transfusions, 7 patients received 5 SDP transfusions, and 2 patients each received 6 and 7 SDP transfusions. Of these 62 transfusion who received more than 1 transfusion, 28 patients were refractory to SDP transfusions. Of these 28 patients $16(57.14 \%)$ were male and $12(42.86 \%)$ were female. Various risk factors associated with refractoriness are fever $(60.71 \%)$, chemotherapy (46.43\%), bleeding (50\%), drug therapy $(42.86 \%)$, splenomegaly $(10.71 \%)$ and others (64.29\%). Out of the 28 patients, 23 patients had no evidence of refractoriness after 1-hour post transfusion, but the 24-hours CCI (Corrected Count Increment) confirmed refractoriness. Both the 1-hour and 24-hour CCI were in refractory range in the rest 5 patients. These 5 patients have been grouped under immune category.

Keywords: Platelet refractoriness; CCI; SDP

\section{Abbreviations: CCI: Corrected Count Increment.}

\section{Introduction}

Platelet refractoriness describes a clinical condition in which patients do not achieve the anticipated platelet count increment from a platelet transfusion. The determination of corrected count increment (CCI) is used to check for patient's platelet count in post platelet transfusion period. The Platelet transfusion refractoriness occurs when there is an inadequate change in the desired level of platelets in the recipient's blood following a platelet transfusion [1]. Corrected count increment can also supplement the determination of a transfusion policy for a patient as also help in checking whether platelet refractoriness has occurred. A corrected count increment more than $7500 \mathrm{~m}^{2} /$ $\left(\mu \mathrm{L} \times 10^{11}\right)$ [although the actual units of the CCI are $\mathrm{m}^{2} /(\mu \mathrm{L}$ $\mathrm{x} 10^{11}$ ), as an index it is typically written without units] at 1-hour and more than 4500 at 24 -hour post transfusion is considered to be an indicator of adequate platelet availability after transfusion.

Inadequate corrected count increment results from the low survival time of platelets due to different immunogenic or non-immunogenic factors. In case of non-immune cause initial increase in the platelet count is seen, while there will be delayed ( $8 \mathrm{hr}$ or $12 \mathrm{hr}$ ) fall in the platelet count, whereas an immune cause will be reflected by a smaller immediate 
increase in the platelet count and marked fall after 24 hours. Correct identification of cause of inadequate corrected count increment will be beneficial to the patient for better management of thrombocytopenia by the treating physician. Among immune-related refractoriness, antibodies against HLA and HPA antigens are the most common factor. Nonimmune factors include splenomegaly, fever, and recent chemotherapy [2].
Aim of the present study is to analysis of platelet refractoriness in patients who received Single Donor Platelets.

\section{Materials and Methods}

The study was conducted in the Department of Transfusion Medicine at IMS and SUM hospital, a tertiary healthcare centre in Eastern India.

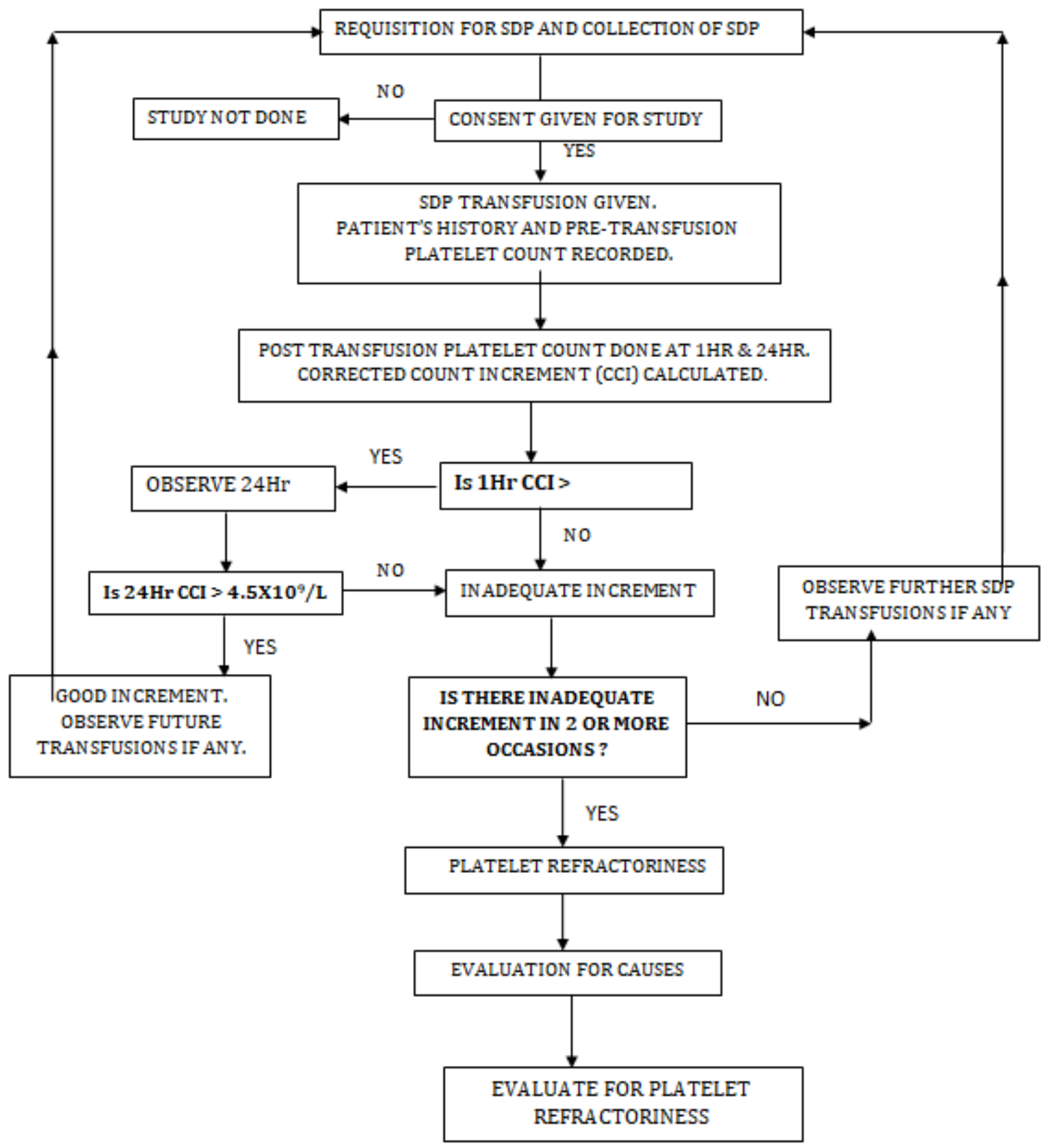

Figure 1: Requisition for SDP and Collection of SDP. 
The study group included subjects who required single donor platelet concentrate at IMS and SUM hospital during the study period. The study was conducted during the period from September 2016 to August 2018. This was a prospective analytical study. Institutional ethics committee approved the study. Data was collected from 155 patients who were admitted to our hospital and required SDP concentrate. Consent was obtained from all participants. Data pertaining to demographic details, medical history, transfusion history, diagnosis, treatment of the patient, indications for platelet transfusion etc. were collected from the treatment file of the respective patients as well as the SDP records maintained at the blood bank. The methodology followed as per flow chart (Figure 1).

\section{Inclusion Criteria}

a) The patients who were advised for transfusion of single donor platelet concentrate were included in the study. b) The patients who consented to be a part of study.

\section{Exclusion Criteria}

a) The subjects who did not consent to be part of the study or wanted to opt out of the study after giving consent were excluded from the study.

b) The data was then be used to calculate the corrected count increment (CCI).

The corrected count increment was calculated as follows:

$\mathrm{CCI}=($ Post-transfusion platelet count $/ \mu \mathrm{L}$ - Pre-transfusion platelet count $/ \mu \mathrm{L}) \times$ Body surface

(Number of platelets transfused) $\times 10^{11}$

\section{Observations and Results}

A total of 155 patients and 285 SDP transfusions were observed. All demographic data of patients were collected. The relevant data of each patient was entered in the performa and in the master chart and analysed statistically. Out of 155 patients 35 patients were from hematology, 36 were from oncology, 11 were from neonatology, 4 from general surgery, 34 from general medicine and 15 from other specialty (Figure 2). Out of 285 transfusions, 1 hour CCI of less than 7500 constituted 25 cases and more than 7500 constituted 260 cases. Out of 285 transfusions, 24 hour CCI of less than 5000 constituted 90 cases and more than 5000 constituted 192 cases. Out of 155 patients who were given 285 SDP transfusions 28 patients were refractory to platelet increment after 2 consecutive platelet transfusions. The average 1-hour platelet increment in these patients was 7473. The average 24 hour CCI was 3889. While in nonrefractory cases $(n=127)$ the average 1 hour CCI was 11376 . The 24 hour average CCI was 7264.

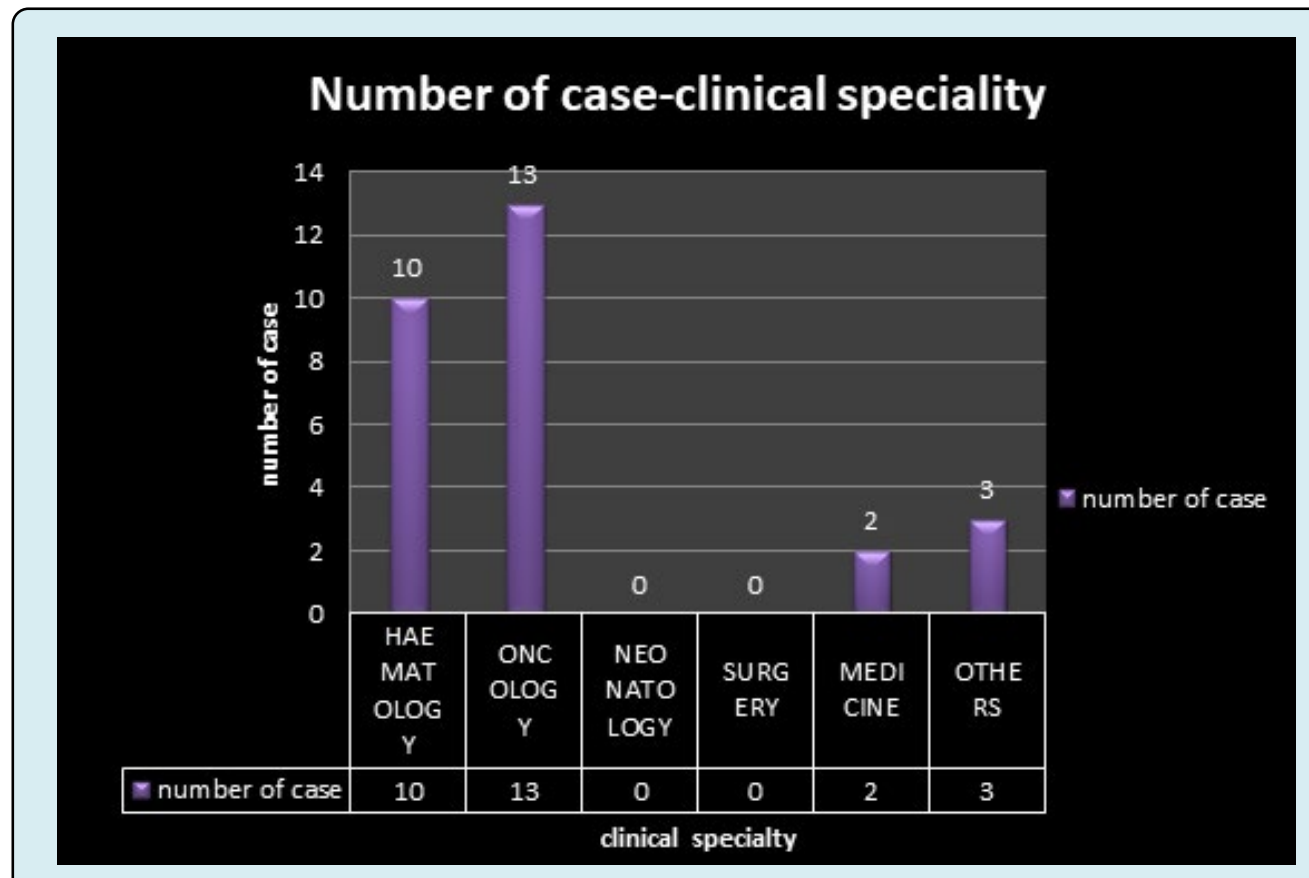

Figure 2: Number of case clinical speciality.

Total 28 cases were found to be refractory, out of which 12 were female and 16 were male. Out of 12 female patients
1 patient was in the age group range of $10-20$ years, 1 in 31 40 years 3 patients in the age group of $41-50$ years, 4 in the 
range of 51-60 years, 2 patient in the years 61-70 years and 1 patient was in the age group range of 71-80 years. Out of 16 male patients 1 was in the age group of 11-20 years, 1 in age-group of 41-50 years, 6 patients in the age group of 5160 years, 4 patients in the age 61-70 years 3 patients were in the age group of 71-80 years and 1 patient was in the age group of $81-90$ years. Out of 28 patients 10 patients were from haematology, 13 were from oncology, 2 from general medicine and 3 from other speciality.

Out of total 28 cases 5 cases showed refractoriness at 1 hour and 23 patients showed refractoriness at 24 hours (Table 1). Of the total 23, 3 patients were having diagnosis of MDS (Mylo-dysplastic syndrome), (Table 2). One patient had ALL( acute lymphoblastic leukemia), 4 patients had diagnosis of CML (Chronic myeloid leukemia), 3 patients had aplastic anemia, 5 patients had AML (acute myeloid leukemia), 1 patient had diagnosis of ITP, 2 patients had CABG, 1 patient was a case of $\mathrm{PPH}, 1$ patient had hypertension and type $2 \mathrm{DM}$, 1 had GI bleed and 1 had myelofibrosis.

\begin{tabular}{|c|c|c|}
\hline Group & No. of Patients & \% of Patients \\
\hline Non Refractory & 127 & 81.9 \\
\hline $\begin{array}{c}\text { 1-HR REFRACTORY } \\
\text { CASES }\end{array}$ & 5 & 3.2 \\
\hline $\begin{array}{c}\text { 24-HR REFRACTORY } \\
\text { CASES }\end{array}$ & 23 & 14.8 \\
\hline Total & 155 & 100 \\
\hline
\end{tabular}

Table 1: Number of Refractoriness.

\begin{tabular}{|c|c|c|}
\hline Disease & 24 Hr Refractory & 1 Hr Refractory \\
\hline MDS & 3 & \\
\hline ALL & 1 & \\
\hline CML & 4 & 1 \\
\hline $\begin{array}{c}\text { APLASTIC } \\
\text { ANAEMIA }\end{array}$ & 3 & 2 \\
\hline AML & 5 & 2 \\
\hline ITP & 1 & \\
\hline POST CABG & 2 & \\
\hline PPH & 1 & \\
\hline HTN T2DM & 1 & \\
\hline GI BLEED & 1 & \\
\hline MYELOFIBROSIS & 1 & \\
\hline TOTAL & 23 & 5 \\
\hline
\end{tabular}

Table 2: Diseases Associated with Platelet Refractoriness.
Various riskfactors wereassociated with the 28refractory patients. Sometime more than 1 risk factor was present in the patient and may be attributable to the development of refractoriness. Fever as a risk factor was associated with 17 patients, while bleeding was associated with 14 patients, chemotherapy was associated with 13 patients, sepsis was associated with 10 patients, splenomegaly was associated with 3 patients, DIC was associated with 3 patients, antibiotics were associated with 8 patients and antiplatelet drugs were associated with 4 patients (Table 3 ).

\begin{tabular}{|c|c|}
\hline Factors & Refractory Patients \\
\hline FEVER & 17 \\
\hline BLEEDING & 14 \\
\hline CHEMOTHERAPY & 13 \\
\hline SEPSIS & 10 \\
\hline SPLENOMEGALY & 3 \\
\hline DIC & 3 \\
\hline ANTIPLATELET DRUG & 4 \\
\hline ANTIBIOTICS & 8 \\
\hline
\end{tabular}

Table 3: Non-Immunological Factors.

Out of total 28 patients 5 patients were associated with one risk factor for platelet refractoriness, 7 patients were associated with 2 risk factors, 12 patients with 3 risk factors, 3 patients with 4 risk factors and 1 patient was associated with 5 risk factors (Table 4).

\begin{tabular}{|c|c|}
\hline No of Risk Factors & Refractory Patients \\
\hline Single & 5 \\
\hline Double & 7 \\
\hline Triple & 12 \\
\hline Four & 3 \\
\hline Five & 1 \\
\hline TOTAL & $\mathbf{2 8}$ \\
\hline
\end{tabular}

Table 4: Risk Factor Association.

\section{Discussion}

Platelet refractoriness occurs in approximately 30 to $70 \%$ of patients who receive multiple transfusions [3]. Main reasons are either clinical factors such as fever, sepsis, splenomegaly, DIC, drugs (e.g. vancomycin, amphotericin B) or immune mediated destruction of platelets $[4,5]$. In present study out of the total 155 patients, 35 patients were from haematology, 36 were from oncology, 11 were from neonatology, 4 from general surgery, 34 from general medicine and 15 from other speciality (Figure 2). Most 


\section{Haematology International Journal}

of the studies concentrated on patients of oncology and haematology.

Out of 155 patients who were given 285 SDP transfusions, 28 patients were refractory to platelet increment after 2 consecutive platelet transfusions. The average 1 hour platelet increment in these patients was 7473 . The average 24 hour CCI was 3889 . While in non-refractory cases $(n=127)$ the average 1 hour CCI was 11376 with maximum increment being 18580 and minimum increment being 7533 . The 24 hour average CCI was 7264 with maximum increment being 23510 and minimum increment being 4281 . Total 28 cases were found to be refractory, out of which 12 were female and 16 were male. Five males and 3 females were A Posititve, 3 males and 5 females were B Positive, 1 female was from 0 negative while 8 male patients and 3 female patients were from 0 Positive. Out of 28 patients 10 patients were from haematology, 13 were from oncology, 2 from general medicine and 3 from other speciality. In the study conducted by Shastry and colleague nine out of 40 patients were found to have refractoriness to platelet transfusion [6]. Out of nine 4 were male and 5 were female. The mean age of patients was 33 years (SD: 17.91 range 10-73).

In the study done by Legler, et al. 274 patients were transfused with SDP concentrate; of these 145 patients received more than two platelet transfusions and were considered for the study regarding the incidence of platelet refractoriness [7]. Out of the above 145 patients, 40 patients (27.6\%) showed refractoriness to platelet transfusions on at one occasion.

In the study done by Slichter, et al. out of the 528 patients observed, $143(27 \%)$ became refractory to platelet transfusion [8]. In study done by Mangwana, et al. 4 patients out of total 30 patients became refractory [9]. In the study done by Kumawat and colleagues out of 30 patients who received platelet transfusions 17 became refractory to platelet transfusions [3]. Klingemann, et al. had reported $34 \%$ (71 of 210) aplastic anaemia patients, refractory to nonleucoreduced pooled random donor platelet transfusions [10]. Out of total 28 cases 5 cases showed refractoriness at 1 hour and 23 patients showed refractoriness at 24 hours. Out of 23 cases 12 were male and 11 were female. Various risk factors were associated with the 28 refractory patients. Sometime more than 1 risk factor was present in the patient and may be attributable to the development of refractoriness (Table 3). Fever as a risk factor was associated with 17 patients, while bleeding was associated with 14 patients, chemotherapy was associated with 13 patients, sepsis was associated with 10 patients, splenomegaly was associated with 3 patients, DIC was associated with 3 patients, antibiotics were associated with 8 patients and antiplatelet drugs were associated with 4 patients. Out of total 28 patients
5 patients were associated with one risk factor for platelet refractoriness, 7 patients were associated with 2 risk factors, 12 patients with 3 risk factors, 3 patients with 4 risk factors and 1 patient was associated with 5 risk factors. (Table 4).

Shastry, et al. in their study had observed 9 refractory patients out of which 3 had aplastic anaemia [6]. The diagnosis done in the other patients were: chronic liver disease, CML, coronary bypass surgery, AML, NHL and viral encephalitis. Splenomegaly was detected in 4 patients, fever found in 5 patients, sepsis found in 4 patients, bleeding observed in 6 patients and DIC found in 4 of the patients. On analysis, it was found that the presence of splenomegaly and administration of antiplatelet drugs had significant contribution to refractoriness. Fever, sepsis, bleeding, DIC and use of cyclophosphamide did not have statistically significant effect on the outcome. Immunological factors though contributed to platelet refractoriness, but had relatively minor contributions.

Kumawat and colleagues in their study had 15 refractory patients out of 30 total patients [3]. Among 15 patients, both immune and nonimmune factors were implicated, whereas alloimmunization and nonimmune factors alone contributed to one case each. Association of HLA class I antibody, HPA antibody to platelet transfusion refractoriness was found significantly associated with refractoriness Nonimmunological causes which were associated significantly with refractoriness were bleeding, infection, and fever. Mangwana, et al. in their study had 4 refractory patients out of 30 patients [9]. Out of four cases three cases, were of myeloid leukaemia; both acute and chronic and one case was of solid organ malignancy in a female (carcinoma ovary). All leukaemia cases were males.

According to Slichter SJ and colleagues 143 patients became refractory [8]. Adverse factors were significantly associated with an increased risk of refractoriness to platelet transfusions and significant among them were lymphocytotoxic antibody positive status, male gender, multiparous status, fever, bleeding, heparin administration and higher weight. Legler TJ, et al. in their study had 40 refractory patients [7]. Non-immune factors, among which most predominant ones were fever and sepsis, were the cause of refractoriness in about $62.5 \%$ of patients. Alloimmunization alone was cause of refractoriness in seven $(17.5 \%)$ of the patients.

In present study out of 62 patients who received 2 or more transfusions 28 patients came out to be refractory which is $45 \%$ of the total study . Kumawat, et al. have similar percentage of refractory patients that is $53.4 \%$ [3]. Shastry and colleagues had $22.5 \%$ refractory patients [6] while Legler, etal. had 27.6\% refractory patients [7]. Mangwana and 


\section{Haematology International Journal}

colleagues had $13.33 \%$ refractory patients [9]. Klingemann, et al. had reported 34\% (71 of 210) aplastic anaemia patients, refractory to non-leucoreduced pooled random donor platelet transfusions [10]. Slichter, et al. reported $27 \%$ patients (143 of 528) refractory to platelet transfusions [8].

Out of 28 patients who were refractory to platelet transfusion 5 were having refractoriness at one hour which tends to be more towards immunological cause which represents $17 \%$ of the total refractory cases. Doughty et al. found that $88 \%$ of 116 refractory transfusions were caused due to non-immune factors [11] which were similar to the results of Shastry, et al. [6] and Kumawat, et al. [3].

\section{Conclusion}

Total of 155 patients and 285 SDP transfusions were observed. The study was done to observe the CCI in patients transfused with SDP concentrates and observe the incidence of refractoriness in these patients. Out of 155 patients 93 patients received 1 SDP concentrates so they were not observed for development of refractoriness. Out of rest 62 patients 28 developed refractoriness. Five patients had a CCI of less than 7500 post 1 -hour while others had CCI more than 7500 post 1 -hour but less than 5000 after 24 hours. Various risk factors were associated with the development of nonimmunological refractoriness such as fever, sepsis, bleeding, splenomegaly, chemotherapy, drugs etc.

\section{References}

1. Murphy MF, Stanworth SJ, Estcourt L (2016) Thrombocytopenia and platelet transfusion. Rossi's Principles of Transfusion Medicine 26: 235-244.

2. Colman RW (2006) Hemostasis and thrombosis: basic principles and clinical practice. Lippincott Williams \& Wilkins.

3. Kumawat V, Sharma RR, Malhotra P, Marwaha N (2015) Prevalence of risk factors for platelet transfusion refractoriness in multitransfused hemato-oncological patients at tertiary care center in North India. Asian J Transfu Sci 9(1): 61-64.

4. Miftode E, Teodor D, Lungu R, Petrovici C (2013) Recurrent thrombocytopenia and Lyme disease. BMC Infect Dis 13(1): P81.

5. Vassallo RR, Murphy S (2010) Apheresis Platelet Collection, Storage, Quality Assessment, and Clinical Use. AABB, Apheresis: Principles and Practices, $3^{\text {rd }}$ (Edn.), pp: 141-165.

6. Shastry S, Chaudhary R (2012) Clinical factors influencing corrected count increment. Transfus Apher Sci 47(3): 327-330.

7. Legler TJ, Fischer I, Dittmann J, Simson G, Lynen R, et al. (1997) Frequency and causes of refractoriness in multiply transfused patients. Ann Hematol 74(4): 185189.

8. Slichter SJ, Davis K, Enright H, Braine H, Gernsheimer $\mathrm{T}$, et al. (2005) Factors affecting posttransfusion platelet increments, platelet refractoriness, and platelet transfusion intervals in thrombocytopenic patients. Blood 105(10): 4106-4114.

9. Mangwana S, Simon N (2016) Evaluation of the platelet cross-matching in oncology patients. Global Journal of Transfusion Medicine 1(1): 16.

10. Klingemann HG, Self S, Banaji M, Deeg HJ, Doney K, et al. (1987) Refractoriness to random donor platelet transfusions in patients with aplastic anaemia: a multivariate analysis of data from 264 cases. $\mathrm{Br} \mathrm{J}$ Haematol 66(1): 115-121.

11. Doughty HA, Murphy MF, Metcalfe P, Rohatiner AZ, Lister TA, et al. (1994) Relative importance of immune and non-immune causes of platelet refractoriness. Vox Sang 66(3): 200-205. 